\title{
Genesis: New Media Art Created as a Visualization of Fluid Dynamics
}

$\operatorname{AUTHOR}(\mathrm{S})$ :

Tosa, Naoko; Pang, Yunian; Zhao, Liang; Nakatsu, Ryohei

\section{CITATION:}

Tosa, Naoko ... [et al]. Genesis: New Media Art Created as a Visualization of Fluid Dynamics. Entertainment Computing - ICEC 2017 2017, 10507: 3-13

\section{ISSUE DATE:}

2017

URL:

http://hdl.handle.net/2433/250218

\section{RIGHT:}

This is a post-peer-review, pre-copyedit version of an article published in Entertainment Computing - ICEC 2017. The final authenticated version is available online at: http://dx.doi.org/10.1007/978-3-319-66715-7_1.; This is not the

published version. Please cite only the published version.; この論文は出版社版でありません。引用の際には出版社版 をご確認ご利用ください。 


\title{
Genesis: New Media Art Created as a Visualization of Fluid Dynamics
}

\author{
Naoko Tosa ${ }^{1}$, Pang Yunian ${ }^{2}$, Liang Zhao ${ }^{2}$, and Ryohei Nakatsu ${ }^{3}$ \\ ${ }^{1}$ Institute for Information Management and Communication, Kyoto University, Kyoto, Japan \\ ${ }^{2}$ Shishukan, Kyoto University, Kyoto, Japan \\ ${ }^{3}$ Design School, Kyoto University, Kyoto, Japan \\ tosa@media.kyoto-u.ac.jp, pang.yunian.87r@st.kyoto-u.ac.jp, \\ liangegsais.kyoto-u.ac.jp, ryohei.nakatsu@design.kyoto-u.ac.jp
}

\begin{abstract}
We have been working on the creation of media art utilizing technologies. As an extension of our previous research, this time we have focused on the visualization of behaviors of fluid. This area has been named as "fluid mechanics" or "fluid dynamics" and there have been various researches in this area. As some of the fluid motion look beautiful, there is another research area called "visualization of fluid motion."

However most of these results show only stable fluid behaviors and lacks of unstable or in other words unpredictable behaviors that would be substantial for the creation of art. Therefore, to create various unstable or unpredictable fluid behaviors in trials to create artworks, we have introduced several new methods such as usage of dry ice, injection of paints into fluid, and usage of air gun to create an explosive effect. This paper proposes the basic concept of new video art called "Genesis" based on the visualization of fluid dynamics, describes details of the above mentioned three ideas that were introduced to create unpredictable fluid dynamics based phenomena, and then describes details of new media art we have created combining these ideas.
\end{abstract}

Keywords: Fluid Dynamics, High Speed Camera, Air Gun, Dry Ice, Media Art.

\section{Introduction}

We have been creating media art where new technologies play an essential role. Recently we have been interested in the usage of a high speed camera by using which we have found that hidden beauty in various physical phenomena could be revealed. Based on this methodology we have been trying to create new type of media art [1]. In our previous trial, we used viscous fluids such as paints with various colors, gave vibration to the fluids, and made them jumping up. Jumping up paints created beautiful forms that change in a very short time which were shot by a high speed camera and then based on editing of the obtained video a new type of media art was created [2]. Also some of such media art were exhibited in the form of projection mapping [3]. Figures 1 and 2 illustrate several scenes of the artwork called "Sound of Ikebana" we have created 
and also a scene of its projection mapping held at ArtScience Museum in Singapore in 2014.
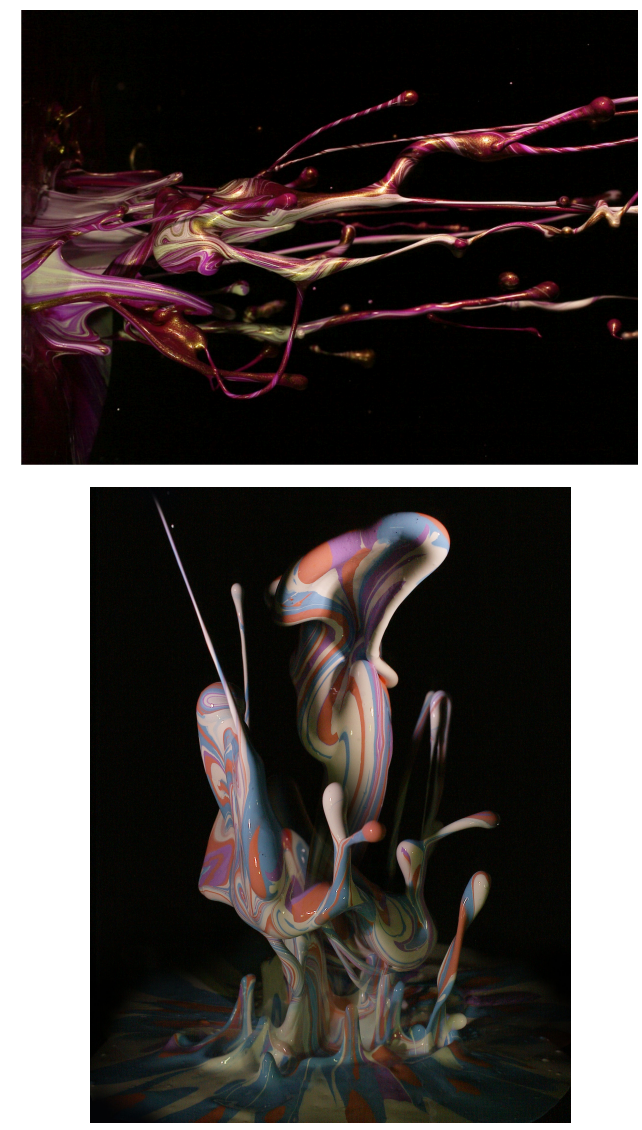

Fig. 1. Several shots of "Sound of Ikebana."

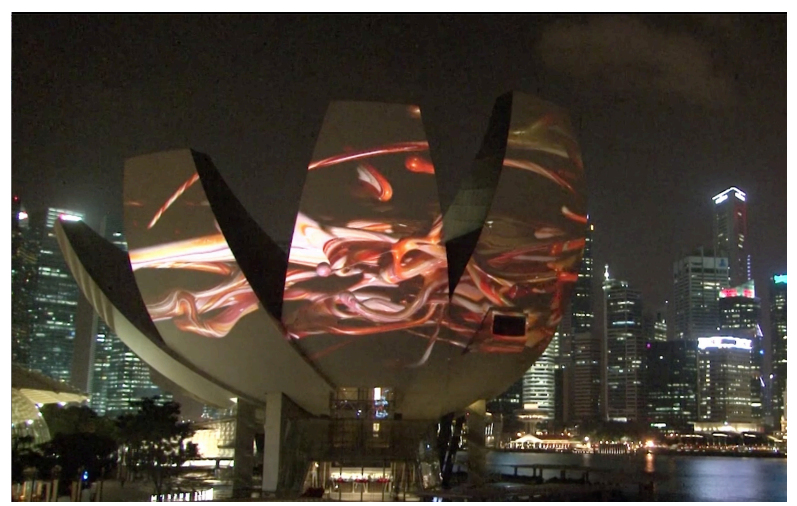

Fig. 2. A scene of the projection mapping of "Sound of Ikebana" carried out at ArtScience Museum in Singapore. 
As an extension of our previous research, this time we have focused on the visualization of behaviors of fluid. This area has been considered as "fluid mechanics" or "fluid dynamics" and there have been various researches in this area [4][5]. As some of the fluid motion look beautiful, there is an another research area called "visualization of fluid motion" [6]. One of such beautiful fluid motions is well known "milk crown" [7].

However most of these results show only stable fluid behaviors and lacks of unstable or in other words unpredictable behaviors that would be substantial for the creation of art. Therefore, to create various unstable or unpredictable fluid behaviors in trials to create artworks, we have introduced several new ideas such as usage of dry ice, injection of paints into fluid, and usage of air gun to create an explosive effect. This paper proposes the basic concept of new video art based on the visualization of fluid dynamics, describes details of the above mentioned three ideas that were introduced to create unpredictable fluid dynamics based phenomena, and then describes details of new media art we have created combining these ideas. This paper consists of the following contents. In Section 2, the basic concept of visualization of fluid dynamics as a method to create artworks will be described. In Section 3, the detailed description of three physical phenomena introduced in the visualization process of fluid dynamics to create new artworks will be described. In Section 4, the details of actual art creation process based on the combination of these three physical phenomena will be described. And in Section 5 conclusion and future work of the paper will be discussed.

\section{Visualization of Fluid Dynamics as a Method to Create Media}

\section{Art}

Study of behaviors of fluid, especially in an environment where several obstacles exist in the pathway of fluid has been a long time research topic in physics and this area has been called as "fluid dynamics" [4][5]. In physics, fluid dynamics is a sub-discipline of fluid mechanics that deals with fluid flow - the science of fluid (liquids and gases) in motion. It has several sub-disciplines itself, including aerodynamics (the study of air and other gases in motion) and hydrodynamics (the study of liquids in motion). Fluid dynamics has a wide range of applications including calculating forces and moments on aircraft, determining the mass flow rate of petroleum through pipelines, predicting weather patterns, understanding nebulae in interstellar space and modeling fission weapon detonation. How to explicitly show the behavior of fluid is another research area called "visualization of scientific phenomena" [6]. Based on this visualization process it became possible for people to watch the actual process of fluid behavior and it has been recognized that various beautiful fluid motion can be created depending on various conditions. As beauty is the fundamental concept of art, utilizing fluid dynamics as a method to create artworks has been one of the key concepts of art creation. There are various artworks that utilize the concept of fluid dynamics. These approaches can be classified into two ways.

One is an approach from purely scientific side. Fluid motion, especially when there are obstacles in the pathway of the fluid, look beautiful and sometimes the visualized result of such fluid motion are considered as art. Figure 3 shows a result of visualization of a stable flow called "laminar flow." As the ratio between inertia and viscosity, called 
"Reynolds number," increases, the laminar flow changes into unstable flow called "turbulence." In turbulence frequently various types of vortex occur, some of which look beautiful. Figure 4 shows one example of such vortex. Other examples are special types of fluid behaviors. One of such behaviors is a well-known phenomenon called "magnetic fluid" or "ferrofluid" which forms itself in specific and beautiful way when it is placed near magnet [7]. Inspired by the beauty of such magnetic fluid several artists have been trying to create artworks. Figure 5 shows an example of the phenomenon of magnetic fluid.

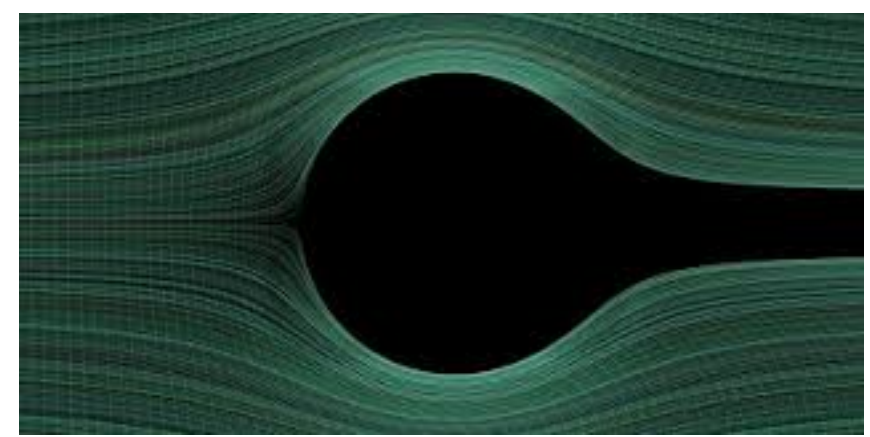

Fig. 3. An example of laminar flow.

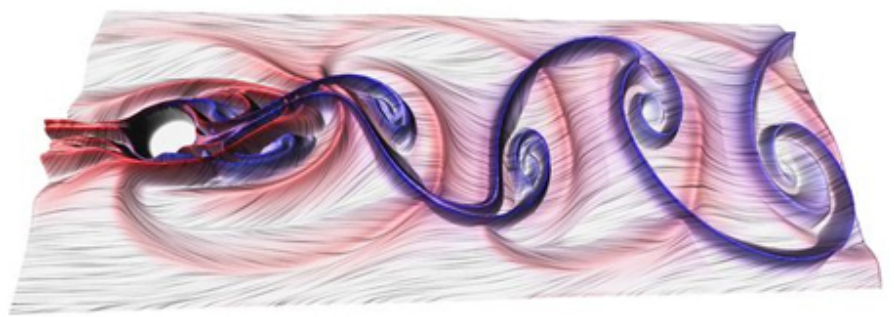

Fig. 4. An example of vorlex.

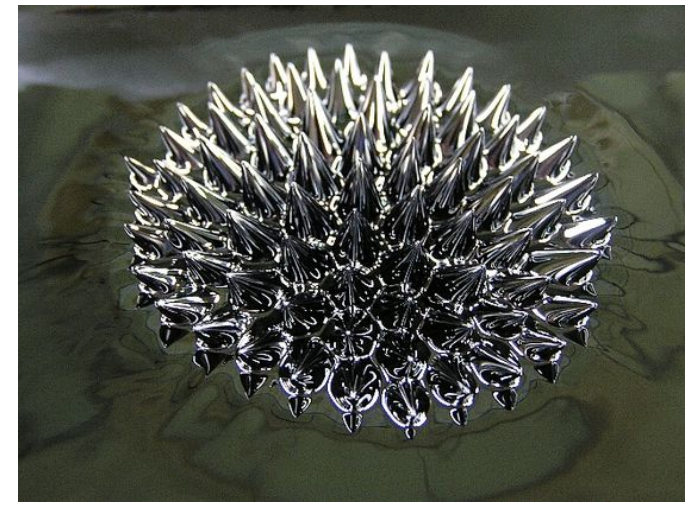

Fig. 5. An example of magnetic fluid. 
Although various types of beautiful forms can be created based on such approaches, created forms based on such approaches are not considered as pure art. The reason is that these phenomena or created forms are still too much physics-based and it is difficult to include in the form creation process "intention of artists." There is a clear distinction between physical phenomenon and art works and the border is how much intention of the artists to create artworks is involved in the created work. If there is no intention or such intention is too weak, created forms are considered physical phenomenon rather than art works. In other words, forms created as physical phenomenon are controlled by laws of physics and there is only a small space where something unexpected happens. And this unexpectedness is very important core part of art works.

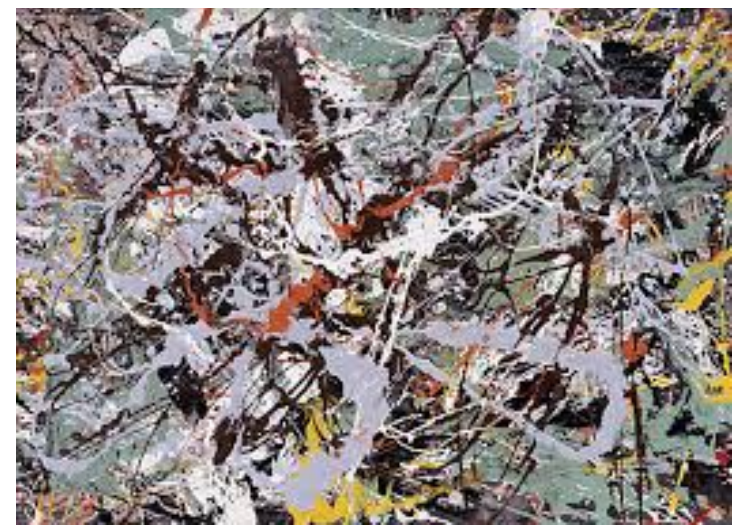

Fig. 6. One of Jackson Pollac's drawings.

On the other hand, there is different approach from art side. Here the usage of fluid is strongly controlled by artists and unexpected phenomena or chance phenomena that happen in the process of fluid usage is utilized by the artists to include something unexpected into their art works. One representative such art creation processes is "Action Painting" [10] led by Jackson Pollock [11]. Action painting is a form of art creation in which, instead of drawing paintings using paintbrush, artists throw or draw paints on a campus. Basically artists have intentions of what kind of paints they use and where on the campus they throw or draw pains. Therefore, in addition to the intensions of artists, a kind of contingency caused by thrown or drawn paints influence the final form of the created artwork. Figure 6 shows one of the representative artwork of Jackson Pollock. Although he is highly evaluated and appreciated in modern history of art, a problem with his art works is that the lack of pure beauty, which has been long time a core concept of great art, and therefore which has confused many people.

Based on the problems included in these two approaches, we think that there should be another way of new art creation somewhere in between these two approaches. We have started from the former approach but tried to include more unexpectedness in created forms. In the next Section several methods to realize this will be described. 


\section{Physical Phenomena Introduced to Realize Unexpected Behavior of Fluid}

We have introduced three new methods into fluid dynamics to realize unexpected behaviors of fluid; namely "injection of paints into fluid," "usage of dry ice as obstacles in fluid pathway," and "usage of air gun to generate explosive effect."

\subsection{Injection of paints into fluid}

As a basic material to observe behaviors we chose color paints. In our previous work to create artworks also we chose color paints and succeeded in creating various types of beautiful and mysterious forms by giving vibrations to them [1][2][3]. And therefore we are familiar with behaviors of color paints. This time, instead of giving them sound vibrations, we tried to inject them into water. Based on various preliminary experiments we found that color paints injected into water from droppers can create interesting forms that resemble the phenomenon of volcano eruption or something similar (Fig. 7).

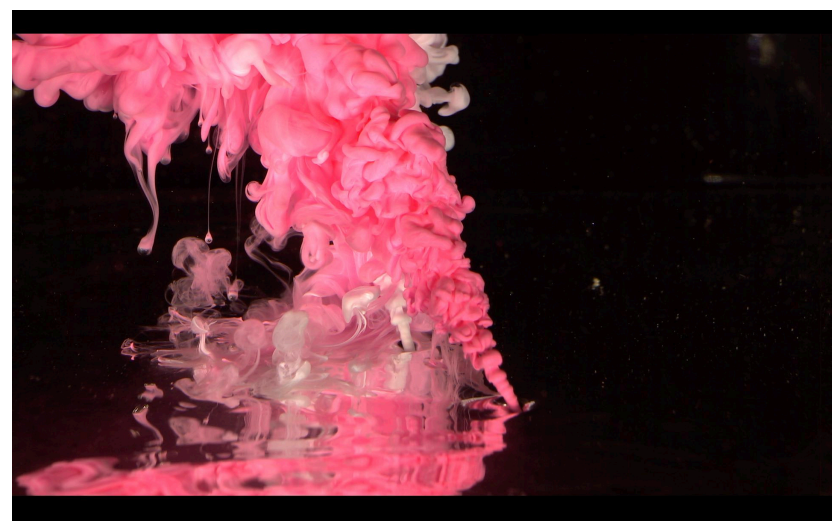

Fig. 7. Injection of color paints into fluid .

As water and color paints even in the case of oil-based pains there is some affinity and threrefore injected paints and water are mingled rapidly and the whole water became a kind of "colored water" rapidly. As what we want to create is interesting behaviors of injected paints, this rapid mingling process is not preferable. Then we tried to add agar into water to increase its viscosity. And we have found that in the case of water with certain amount of viscosity has the effect of delaying such mingling process. Also we have found that level of viscosity based on the amount of added agar plays an important role by changing the mingling time to some extent. This finding was important to create interesting behavior of injected paints. However, basically behaviors of injected paints are based on diffusing process and, as the time passes, water and paint are mixed based on one-way process. Therefore it is difficult to generate something unexpected based on this basic method. Therefore, some new mechanism of creating unexpected phenomenon should be introduced. To realize this, we have introduced the usage of dry ice which is described in next subsection. 


\subsection{Usage of dry ice as obstacles in fluid pathway}

Based on fluid dynamics study we have learned that the existence of obstacles in the pathway of fluid motion is the key to generate beautiful and mysterious forms. At the same time, we have learned that such obstacles should not be fixed ones. Fixed obstacles gives fixed effects to the behaviors of fluid and this process is not effective in generating something unexpected. Therefore, such obstacles should move around. Also it is preferable that the moving patterns of such obstacles would be unstable or even unexpected. In addition, it would be preferable if forms of the obstacles would change unexpectedly. We have carried out various kinds of experiments to find out such obstacles and finally found that the use of dry ice is very effective to be used as obstacles to be interacted with injected paints.

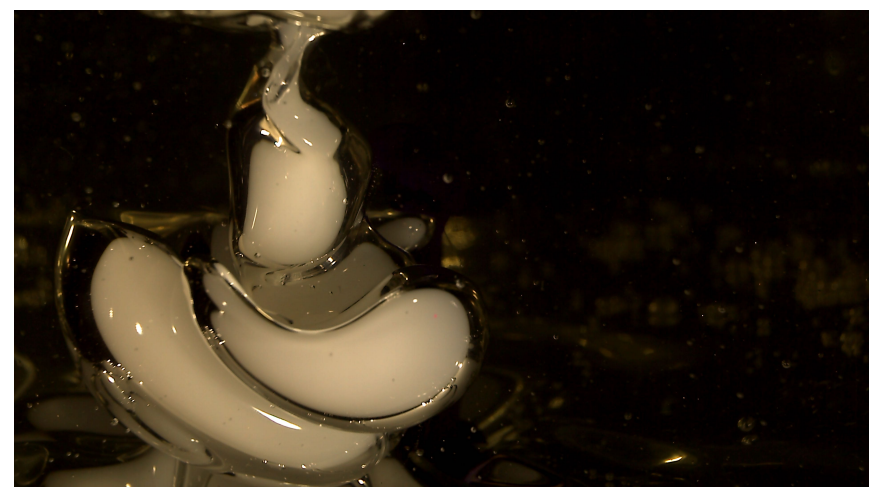

Fig. 8. Bubbles generated by dry ice.

Dry ice is the solid form of carbon dioxide. It is used primarily as a cooling agent. Its advantages included lower temperature than that of water ice and not leaving residue. At the same time, dry ice has been frequently used as a material to create mysterious stage effect, as it creates huge amount of fog when it is put into water. People have been focusing on the effect of fog generation when they use dry ice. However, we have focused on the early process of fog generation. When dry ice is put into water, based on the temperature difference between water and dry ice, rapid vaporization process of dry ice occurs. Many small bubbles, each of which contains carbon dioxide fog, are generated as the result of vaporization and these small bubbles rise from dry ice at bottom to water surface and finally create fog. Watching this process by using a high speed camera, we have found that such bubbles have interesting forms with each bubble having a different form. And during the process of its rising up to water surface the bubble always changes its form. This phenomenon gives us an impression that each bubble is a kind of living creature (Fig. 8). Then we had an idea that the combination of these bubbles and injected paints described in the previous subsection would be ideal to generate a new type of phenomenon based on fluid dynamics. Therefore, we have adopted the usage of dry ice as an obstacle material in the pathway of injected fluid.

\subsection{Usage of air gun to generate explosive effect}

Based on our observation we have found that each of the two described effects and especially their integration could create really new fluid dynamics based effect that would be described in detail in the next section. At the same time, we have found that it would be important and necessary for us to have another phenomenon that can create 
surprising effect such as an explosive effect. In our previous work we used an air gun to explode frozen flowers and this could create really beautiful and at the same time surprising effect [3]. Based on our experience we have decided to use again an air gun to realize a new effect. Based on various kinds of experiments, we have found that shooting an air gun bullet into water could create a surprising phenomenon. By being shot into water with relevant speed, firstly an air gun bullet creates a big hollow ball with nothing inside. Then the ball crashes itself quickly by intriguing surrounding water as if there is an internal waterfall in water. This process is difficult to watch by our naked eye but is observable once the process is shot by a high speed camera. This process is shown in Fig 9 and 10 and because of an amazing effect it can create, we have decided to adopt it in combination with the above mentioned two methods.

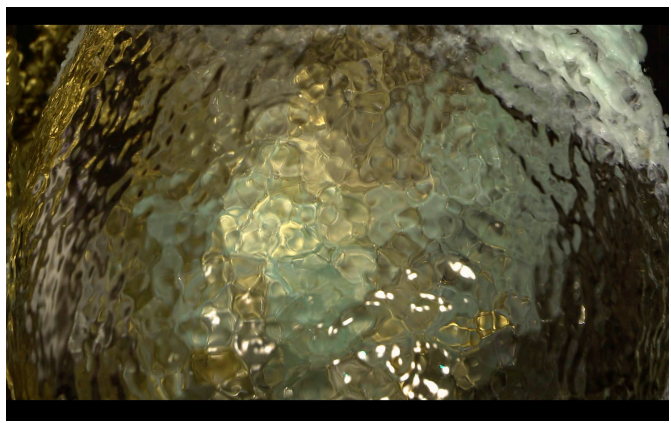

Fig. 9. Effect of an air gun bullet shot into fluid.

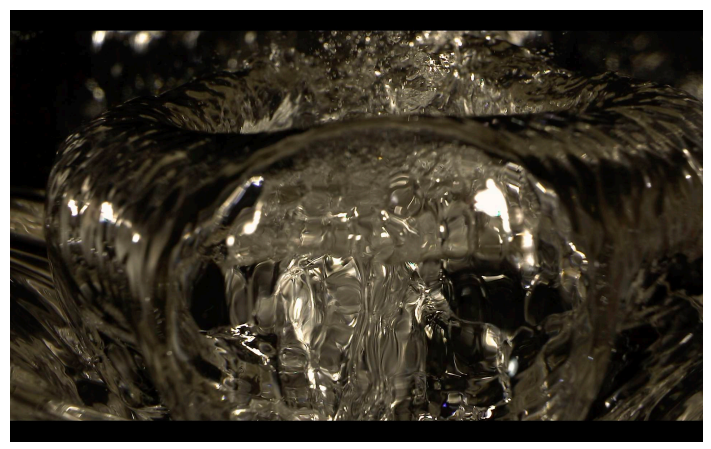

Fig. 10. Break down of a bubble created by an air gun bullet.

\section{Physical Phenomena Introduced to Realize Unexpected Behavior of Fluid}

By combining three types of new effect creation methods described in the previous Section, we tried to create new type of physical phenomena. As a first step, we have combined two methods described in subsections 3.1 and 3.3. The actual process is as follows. Firstly, combination of color paints is selected. Of course single color is acceptable. But combination of several colors is more effective as it can generate beautiful and deep effect. At the same time, an air gun bullet is shot into water aiming the injected color paints. As this whole process occurs in a very short time, this process 
can become visible only by being shot using a high speed camera. Several examples of obtained images are shown in Figs. 11. We have found that the water falling effect, that occurs when a hollow bubble created by a shot bullet, gives us the feeling that comic dust is being drawn into a black hall. This is the main reason that we have decided to call our new artwork as "Genesis" which means the end and also reborn of our cosmos, life, etc.
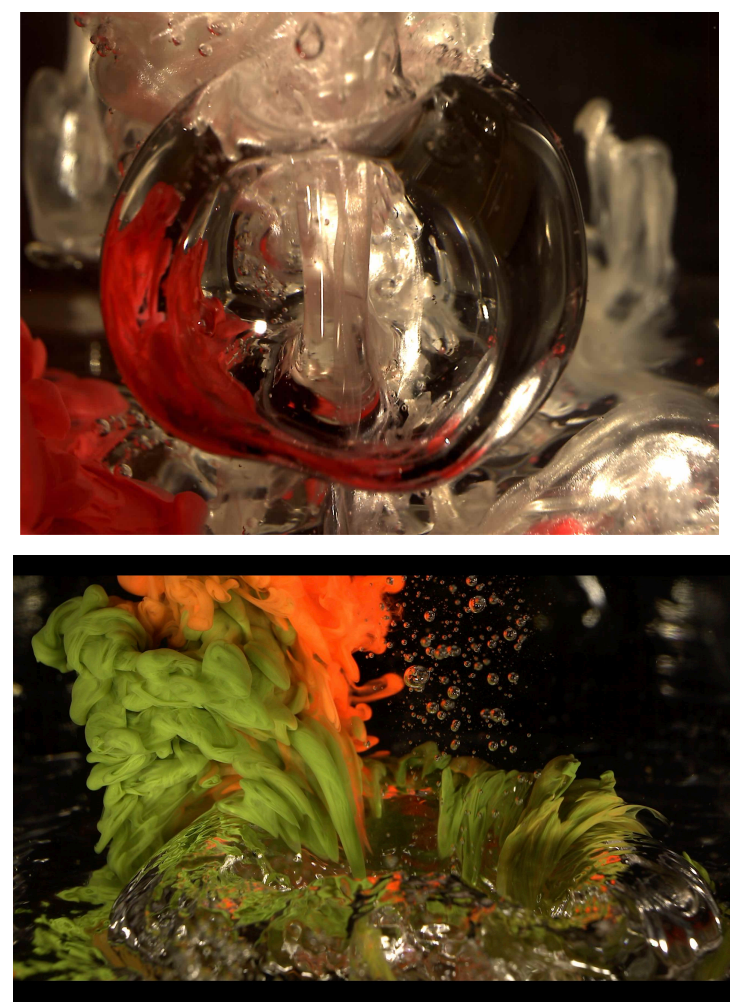

Fig. 11. Several examples of interaction between a bubble created by an air gun bullet and injected color paints.

As a next step we tried to integrate two methods described in subsections 3.1 and 3.2. Firstly, we put a small block of dry ice into water letting it generate bubbles with carbon dioxide fog inside. Then a combination of several color paints is injected into water. Without dry ice based bubbles, the injected color paints quickly diffuse making the whole water as a colored water. There are two ways to avoid this somewhat boring event to happen. One is that, as is described in subsection 3.1, we have used agar to increase water viscosity to some extent. Based on several experiences we have found that there is a certain range of viscosity in which the diffusion of color paint into water occurs slowly. Then under such condition we added dry ice into water. As described in subsection 3.2, various dry ice bubbles are generated as the result of vaporization process of dry ice where forms of dry ice bubbles are different each other and even their forms change continuously while rising up in water to water surface. Then injected color paints interact with these various bubbles and create various complex forms as shown in Fig. 12. These created forms are beyond the forms we often see as the result of scientific 
visualization and look very artistic. Combining images obtained in the first step and the second step can become sophisticated media art that has never been created.
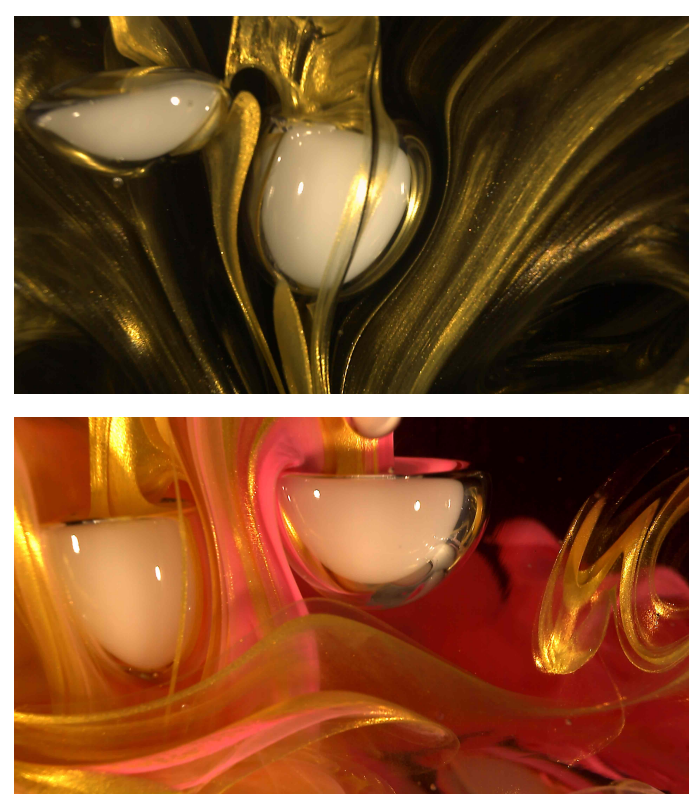

Fig. 12. Several examples of interaction between bubbles created by dry ice and injected color paints.

\section{Conclusion}

In this paper we have proposed a new type of media art creation method and described its details. As an extension of our previous experience of creating media art based on the movement of color paints that are vibrated and jumped up forming various types of surprising and mysterious forms, we have tried to explore different type of fluid dynamics based phenomenon. This time we have decided to explore the behavior of color paints in water. To give people different type of sense of surprise and mystery we have introduced three methods to create new fluid dynamics phenomenon. One is an effect obtained by injecting color paints into water with various viscosity. Second is to use dry ice as obstacles that would interact with the flow of injected paints and based on this to create surprising and mysterious liquid forms. In addition to these phenomena, we have decided to use an air gun and we have found that an air gun bullet shot into water could create a new type of explosive effect. By integrating these three methods in a relevant way and also by using a high speed camera to shoot and visualize generated phenomena that occur in very short time, we have tried to create various kinds of new phenomena. Interestingly enough what we could create is something beautiful, noble, and inspiring that never existed and that is somewhere between existing two types of art creation process. One is a purely scientific process and its aim is to find out beauty in the process of liquid motion as a physical phenomenon. Another is the usage of liquid as a basic material for creating drawing. Here the basic idea of art creation is controlled by an artist but in the final art making process of paint throwing and dropping a 
randomness, that is one of basic natures of physical phenomena, is included to add values to the created artwork.

We have found that our proposed method situates somewhere in between these two different processes. Its feature is that on one hand it can keep pure beauty included in physical phenomenon that is something missing in the art creation process called Action Painting. On the other hand, our method can get rid of a feeling associated with too scientific phenomena which we would have when watching works created purely based on uncontrolled physical phenomenon. Therefore, we believe that we succeeded in creating a new type of artwork. We are going to exhibit our newly created artworks at various exhibitions over the worlds to know responses from people in different countries and cultures. We hope and expect that our new artwork would be welcomed by people in various countries with different cultures.

\section{Reference}

1. Feng Chen, Tomoji Sawada, Naoko Tosa: Sound Based Scenery Painting. 2013 International Conference on Culture and Computing, IEEE Press (2013).

2. Yunian Pang, Naoko Tosa: New Approach of Cultural Aesthetic Using Sound and Image. 2015 International Conference on Culture and Computing, IEEE Press (2015).

3. Naoko Tosa, Ryohei Nakatsu, Pang Yunian, Kosuke Ogata: Projection Mapping Celebrating RIMPA 400th Anniversary. 2015 Conference on Culture and Computing, IEEE Press (2015).

4. Bruce R. Munson, et al.: Fundamentals of Fluid Mechanics. Wiley (2012).

5. Peter S. Bernard: Fluid Dynamics. Cambridge University Press (2015).

6. A. J. Smits, T. T. Lim eds.: Flow Visualization: Techniques and Examples. Imperial College Press (2012).

7. Rouslan Krechetnikov, George M. Homsy: Crown-forming Instability Phenomena in the Drop Splash Problem. Journal of Colloid and Interface Science, Vol.331, No.2, pp.555-559 (2009).

8. Stefan Odenbach ed.: Colloidal Magnetic Fluids: Basics, Development and Applications of Ferrofluid. Lecture Note in Physics, Springer (2009).

9. Sachiko Kodama: Dynamic Ferrofluid Sculpture: Organic Shape-Changing Art Forms. Communication of ACM, Vol.51, No.6, pp.79-81 (2008).

10. Robert Fleck, et al.: Action Painting. Hatje Cantz (2008).

11. Ellen G. Landau: Jackson Pollock. Harry N. Abrams (2010). 